\title{
Attempts to remodel the pathways of gemcitabine metabolism: Recent approaches to overcoming tumours with acquired chemoresistance
}

\author{
Yuriko Saiki ${ }^{1,2}$, Shuto Hirota ${ }^{1}$, Akira Horii ${ }^{1,3}$ \\ 'Department of Molecular Pathology, Tohoku University School of Medicine, Sendai, Miyagi 980-8575, Japan. \\ ${ }^{2}$ Office of Medical Education, Tohoku University School of Medicine, Sendai, Miyagi 980-8575, Japan. \\ ${ }^{3}$ Saka General Hospital, Shiogama, Miyagi 985-0024, Japan.
}

Correspondence to: Drs. Akira Horii and Yuriko Saiki, Department of Molecular Pathology, Tohoku University School of Medicine, 2-1 Seiryo-machi, Aoba-ku, Sendai, Miyagi 980-8575, Japan. E-mails: horii@med.tohoku.ac.jp; ysaiki@med.tohoku.ac.jp

How to cite this article: Saiki Y, Hirota S, Horii A. Attempts to remodel the pathways of gemcitabine metabolism: recent approaches to overcoming tumours with acquired chemoresistance. Cancer Drug Resist 2020;3:819-31.

http://dx.doi.org/10.20517/cdr.2020.39

Received: 3 Jun 2020 First Decision: 2 Jul 2020 Revised: 19 Jul 2020 Accepted: 8 Sep 2020 Available online: 12 Oct 2020

Academic Editor: Nils Brünner Copy Editor: Cai-Hong Wang Production Editor: Jing Yu

\begin{abstract}
Gemcitabine is a cytidine analogue frequently used in the treatment of various cancers. However, the development of chemoresistance limits its effectiveness. Gemcitabine resistance is regulated by various factors, including aberrant genetic and epigenetic controls, metabolism of gemcitabine, the microenvironment, epithelial-tomesenchymal transition, and acquisition of cancer stem cell properties. In many situations, results using cell lines offer valuable lessons leading to the first steps of important findings. In this review, we mainly discuss the factors involved in gemcitabine metabolism in association with chemoresistance, including nucleoside transporters, deoxycytidine kinase, cytidine deaminase, and ATP-binding cassette transporters, and outline new perspectives for enhancing the efficacy of gemcitabine to overcome acquired chemoresistance.
\end{abstract}

Keywords: Gemcitabine, chemoresistance, deoxycytidine kinase, human equilibrative nucleoside transporter 1, cytidine deaminase, ATP-binding cassette transporters, metabolism

\section{INTRODUCTION}

Gemcitabine [2',2'-difluoro-2'-deoxycytidine $(\mathrm{dFdC})]$, was first described by Eli Lilly and Company in $1986^{[1]}$ and is the most important deoxycytidine nucleoside analogue with fluorine substituents at the ${ }^{\prime}$ 


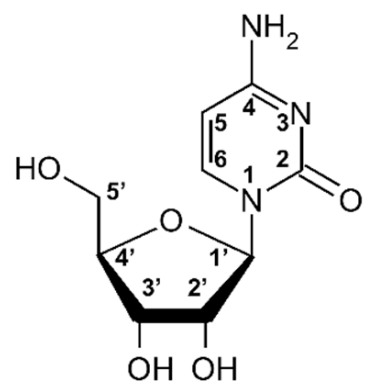

Cytidine

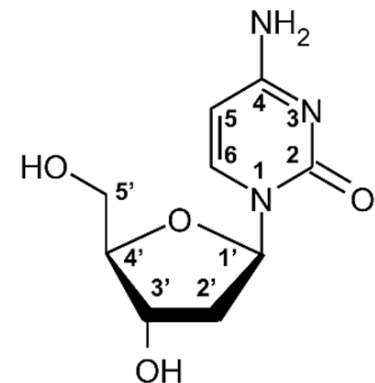

Deoxycytidine<smiles>Nc1ccn(C2O[C@H]3COC[C@H](C3O)C2(F)F)c(=O)n1</smiles>

Gemcitabine

Figure 1. Structures of cytidine, deoxycytidine and gemcitabine

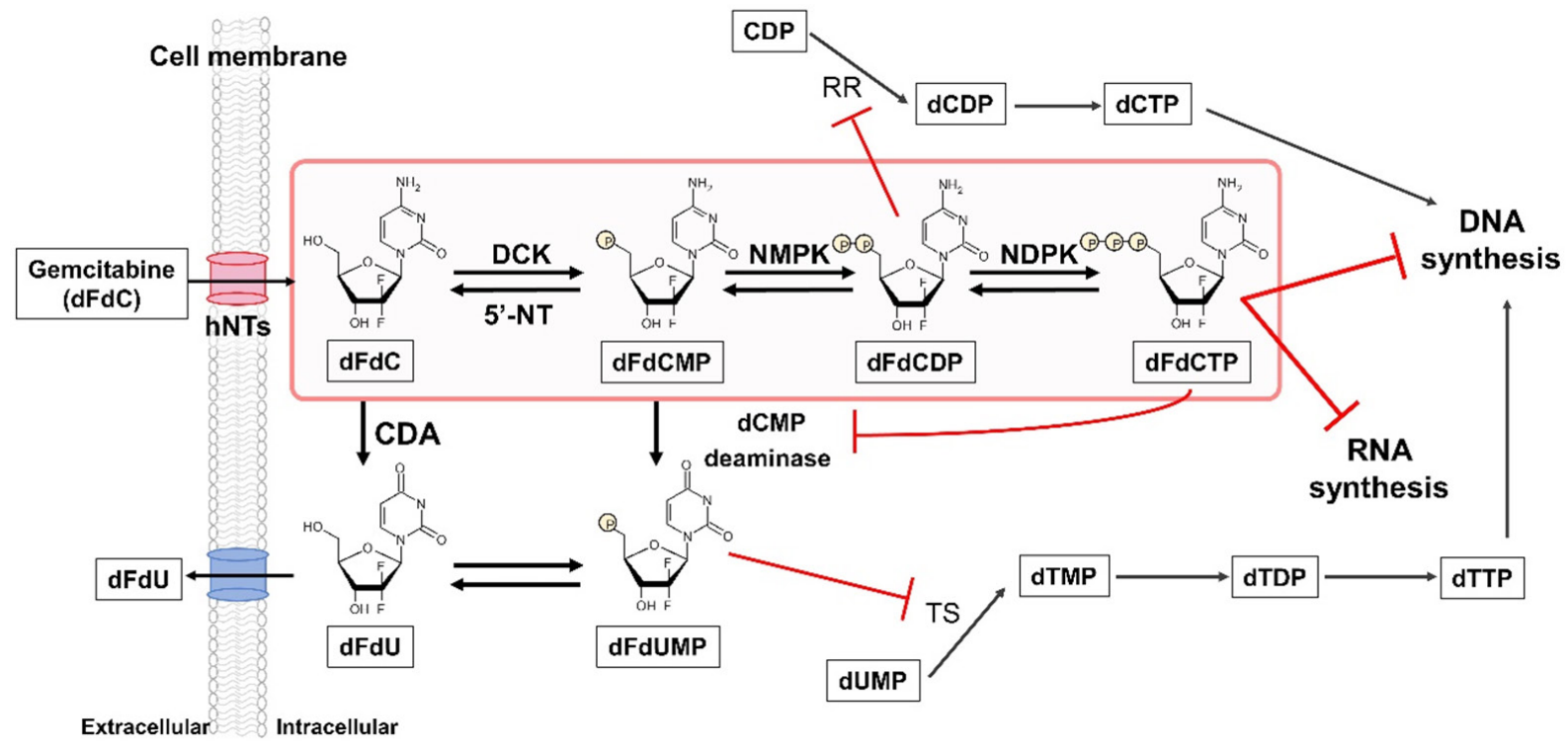

Figure 2. Metabolism and action of gemcitabine [difluoro $2^{\prime}$-deoxycytidine (dFdC)]. dFdC is transported into the cell through nucleoside transporters (hNTs), then stepwise phosphorylated by deoxycytidine kinase (DCK), nucleoside monophosphate kinase (NMPK), and nucleoside diphosphate kinase (NDPK), to form active triphosphate metabolite (dFdCTP). This molecule then inhibits DNA and RNA synthesis. Diphosphate metabolite (dFdCDP) inhibits ribonucleotide reductase (RR), an enzyme that catalyses the conversion of ribonucleotide (CDP) to deoxyribonucleotide (dCDP). The majority of $\mathrm{dFdC}$ is inactivated mainly by cytidine deaminase (CDA) mediated conversion to difluorodeoxyuridine ( $d F d U$ ) and then excreted through the $A B C$ transporter. Deamination of $d F d C M P$ to dFdUMP by deoxycytidylate deaminase (dCMP deaminase) and subsequent dephosphorylation forms dFdU; this is another inactivation pathway of dFdC. dFdUMP inhibits thymidylate synthase (TS), resulting in the depletion of the dTMP pool. dFdCTP inhibits dCMP deaminase

position of the pentose ring [Figure 1] $]^{[2]}$. Its metabolic pathway is illustrated in Figure 2. This molecule is hydrophilic, and can be transported into cells by nucleoside transporters (hNTs), including both sodium-dependent concentrative nucleoside transporters (hCNTs) and sodium-independent equilibrative nucleoside transporters (hENTs). hCNTs mediate unidirectional transportation of nucleosides. hENT1 can uptake gemcitabine with high affinity but low capacity, whereas hENT2 can uptake gemcitabine with low affinity but high capacity. The intracellular uptake of gemcitabine is mainly mediated by hENT1 in cancer cells. In hepatocytes, the uptake of gemcitabine is mainly mediated by low affinity hENT2 ${ }^{[3,4]}$.

Gemcitabine is a prodrug which requires intracellular phosphorylation for activation. Inside the cell, gemcitabine is phosphorylated to its monophosphate form (dFdCMP) by deoxycytidine kinase (DCK) and is then further phosphorylated to its diphosphate ( $\mathrm{dFdCDP}$ ) and then triphosphate forms (dFdCTP), as 
shown in Figure 2. The resulting dFdCTP is incorporated into DNA and then the DNA strand synthesis is terminated after incorporation of another nucleotide, hiding dFdCTP from DNA repair enzymes ${ }^{[5]}$. $\mathrm{dFdCTP}$ is also incorporated into $\mathrm{RNA}^{[6,7]}$, and sensitivity to gemcitabine is related to differences in RNA incorporation ${ }^{[8]}$. RNA incorporation of gemcitabine may play an important role in its activity. dFdCDP is an effective inhibitor of ribonucleoside-diphosphate reductase, an enzyme that transforms CDP into dCDP; this results in a decrease of the dCTP pool. Deamination of dFdCMP by dCMP-deaminase forms dFdUMP. Thymidylate synthase, which plays a key role in the synthesis of thymidine monophosphate (TMP) ${ }^{[9]}$, is another target for gemcitabine, via dFdUMP. The natural substrate of TS, 2'-deoxyuridine monophosphate (dUMP), resembles dFdUMP, and it inhibits TS resulting in a depletion of the TMP pool.

Evidence for the usefulness of gemcitabine as a potent anti-tumour reagent has been reported; it is used either alone, or in combination with other agents for patients with pancreatic ductal adenocarcinoma $(\mathrm{PDAC})^{[10]}$ and several other human cancers, such as non-small cell lung cancer, breast cancer, ovarian cancer, and bladder cancer ${ }^{[11]}$ (approved by FDA). However, acquisition of chemoresistance against gemcitabine significantly limits its effectiveness. Chemoresistance can be divided into two categories, intrinsic and acquired, in the course of drug treatment ${ }^{[12]}$. Activities of drug transporters and metabolizing enzymes have been considered to be strongly involved in the chemoresistance to gemcitabine. Epithelialto-mesenchymal transition (EMT) is not only related to a phenotypic change in the tumour cells; it also contributes to gemcitabine resistance ${ }^{[13]}$. Based on gene expression profiles of pancreatic cancer cell lines, gemcitabine-resistant cells contain many features consistent with $\mathrm{EMT}^{[14]}$. Exosomes have shown to be involved in gemcitabine resistance by delivering miRNAs. Exosomal miR-106b from cancer-associated fibroblasts ${ }^{[15]}$ and miR-210 from cancer stem cells ${ }^{[16]}$ both promote gemcitabine resistance. However, these areas are beyond the focus of this review, and we will discuss the challenges of remodelling the gemcitabine metabolizing pathway to overcome acquired chemoresistance against gemcitabine.

\section{IMPROVEMENT OF GEMCITABINE UPTAKE}

The membrane permeability of gemcitabine is poor in human cells. It is mediated by five distinct hNTs with different affinities; two equilibrative-type (hENT1, hENT2) and three concentrative-type transporters (hCNT1, hCNT2, hCNT3) ${ }^{[17-19]}$. Among these, hENT1 functions as the major gemcitabine transporter; in vitro experiments have demonstrated that increased expression of hENT1 is the critical factor for sensitivity to gemcitabine ${ }^{[20]}$. Restriction of intracellular uptake of gemcitabine by suppressed expression of hENT1 is one of the established mechanisms of drug resistance ${ }^{[19,21]}$. The majority of studies on patients with resected pancreatic cancer have suggested that high expression of this hENT1 may be predictive of improved survival in patients treated with gemcitabine ${ }^{[22-24]}$. Disrupted expression of hENT2 on the plasma membrane causes impaired uptake of gemcitabine, resulting in acquired chemoresistance of pancreatic cancer cells ${ }^{[25]}$.

Currently, several approaches to enhancing the efficacy of gemcitabine uptake or to bypass the hNTs have been introduced. hCNT1 is frequently diminished in pancreatic cancer cells compared with normal pancreatic ductal epithelial cells ${ }^{[26]}$, so drug inhibition or degradation of hCNT1 can increase the transportation of gemcitabine, and thus improve its efficacy ${ }^{[27]}$. A recent study indicated that mucin 4 (MUC4) suppresses hCNT1 expression and that inhibition of MUC4 enhances gemcitabine sensitivity ${ }^{[28]}$.

NEO6002 is a gemcitabine modified cardiolipin [Figure 3A]. This molecule entes the cell independently of $\mathrm{hNT}$, and exerts higher activity, with lower toxic adverse side effects in mouse tumour xenograft model ${ }^{[29]}$. Another lipophilic prodrug, gemcitabine-elaidic acid conjugate CP-4126 [Figure 3A], also known as CO-101, is transported into the cells independently of hENT1 and has been demonstrated to be effective in vitro and in various human cancer models ${ }^{[30]}$. However, a long-term survival analysis found that the survival rate of patients using CP-4126 was not superior to gemcitabine in patients with low expression of hENT1 in 
A

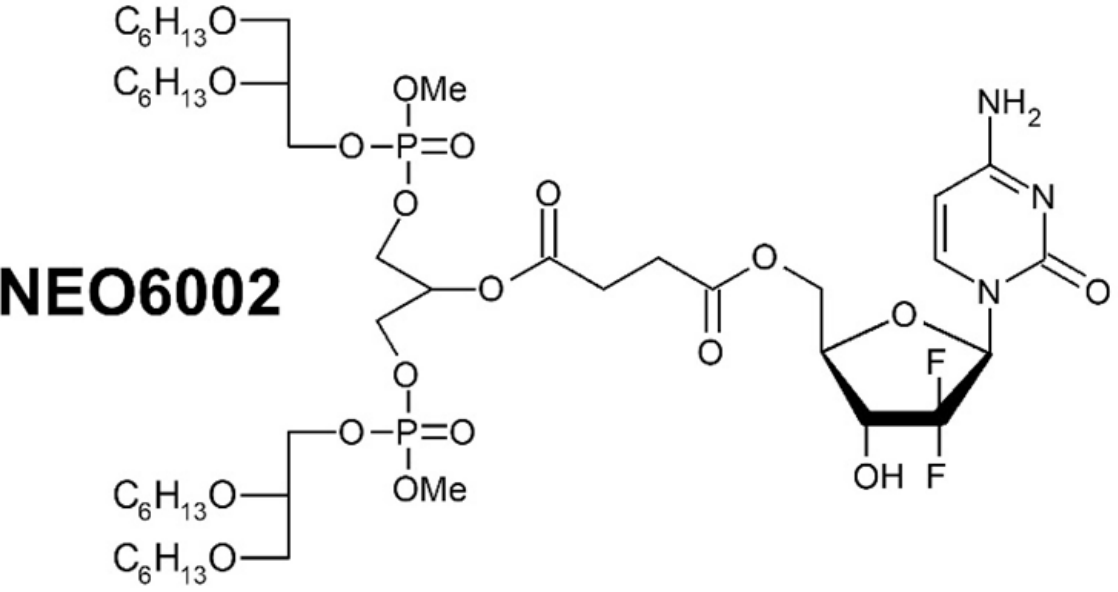

B
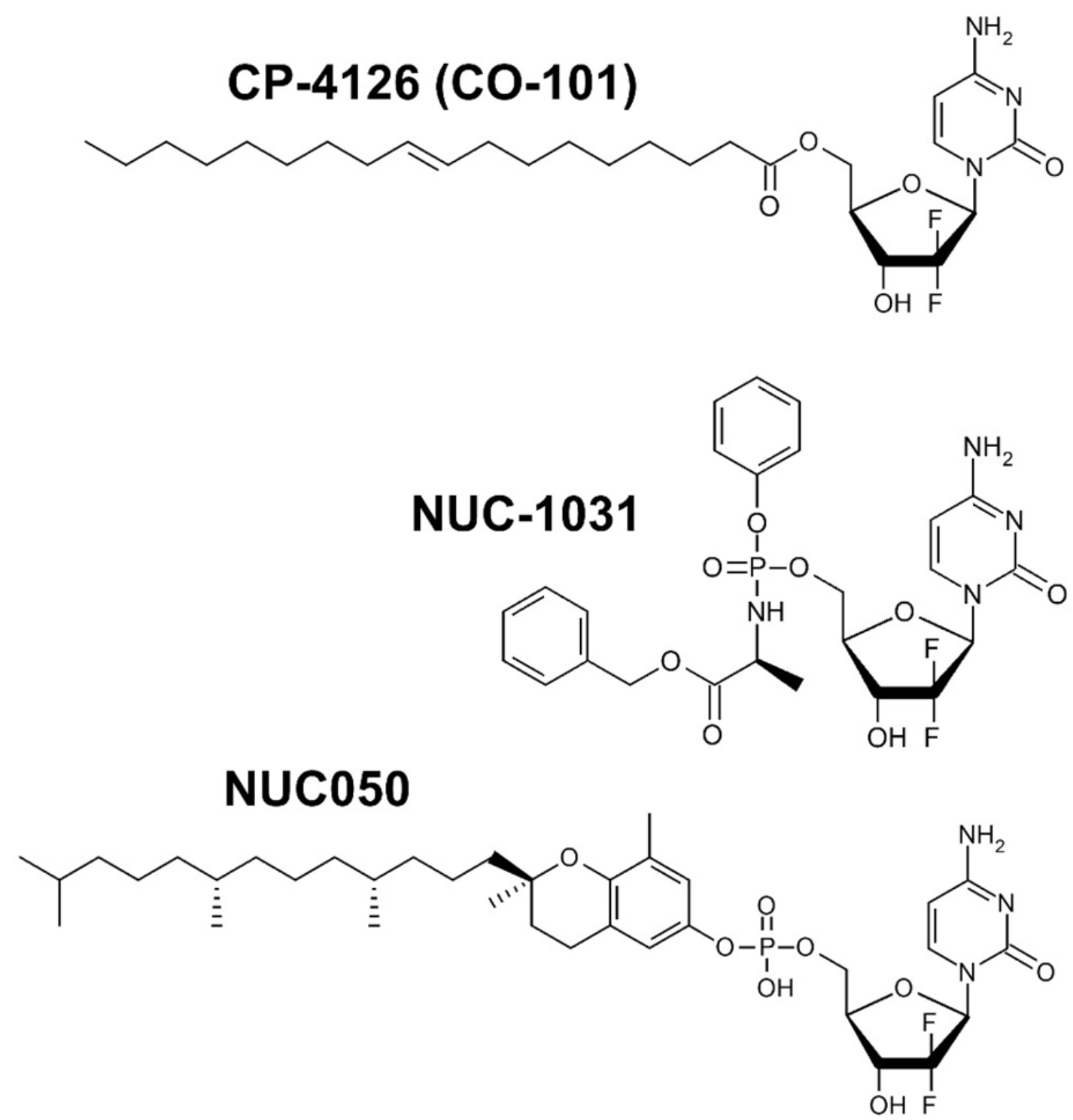

Figure 3. Structures of NEO6002 and CP4126, the gemcitabine-modified compounds that can bypass hNT-mediated introduction inside the cell (A); structures of NUC-1031 and NUC050, gemcitabine-modified compounds that can bypass the deoxycytidine kinasemediated activation pathway of gemcitabine (B) 


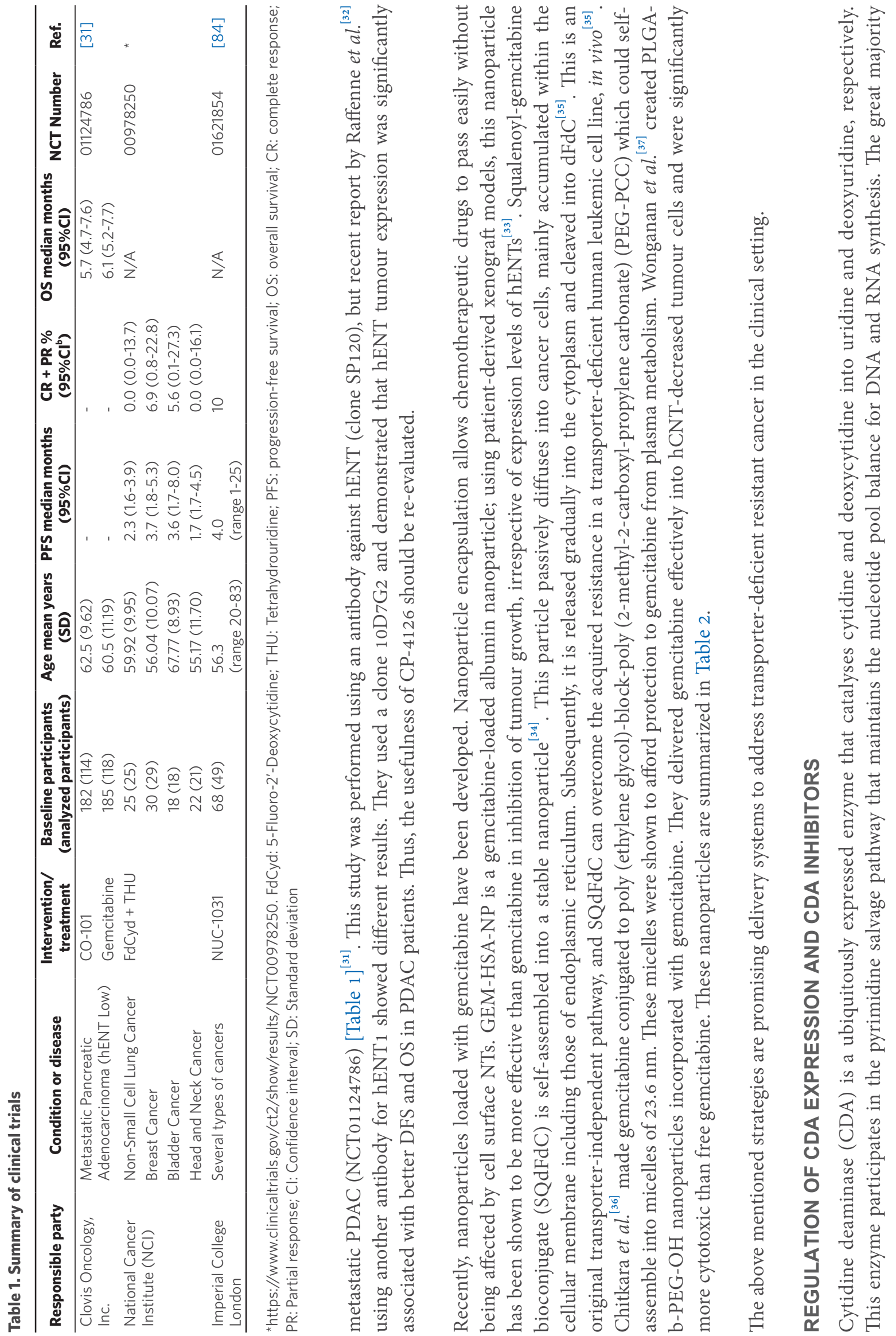


Table 2. Examples of gemcitabine-containing nanoparticles and their effects

\begin{tabular}{|c|c|c|c|c|}
\hline Nanoparticle & Ingredient & & Outcome & Ref. \\
\hline \multirow[t]{2}{*}{ GEM-HSA-NP } & albumin & in vitro & $\begin{array}{l}\text { Inhibited cell proliferation, arrest cell cycle and induced apoptosis in } \\
\text { pancreatic cancer cell lines. }\end{array}$ & \\
\hline & & in vivo & $\begin{array}{l}\text { More effective than gemcitabine when inhibiting tumour growth } \\
\text { whether the expression levels of hENT1 were high or low in PDX models. } \\
\text { The biotoxicity did not increase compared with gemcitabine. }\end{array}$ & {$[33]$} \\
\hline \multirow[t]{2}{*}{ SQdFdC } & squalene & in vitro & $\begin{array}{l}\text { Exhibited superior anticancer activity in human cancer cells and } \\
\text { gemcitabine-resistant murine leukaemia cells. }\end{array}$ & \\
\hline & & in vivo & $\begin{array}{l}\text { Exhibited superior anticancer activity in experimental leukemic mouse } \\
\text { modes both after intravenous and oral administration. }\end{array}$ & [34] \\
\hline \multirow[t]{2}{*}{ PEG-PCC GEM } & PEG-PCC & in vitro & Induced cell apoptosis in pancreatic cancer cell lines & \\
\hline & & in vivo & Significantly inhibited tumour growth in xenograft bearing mice & {$[36]$} \\
\hline PLGA-b-PEG-OH GEM & PLGA-b-PEG-OH & in vitro & $\begin{array}{l}\text { Effectively delivered gemcitabine into hCNT-decreased ovarian cancer } \\
\text { cells and showed significant cytotoxicity compared to free gemcitabine. }\end{array}$ & {$[37]$} \\
\hline
\end{tabular}

hENT1: human equilibrative nucleoside transporter 1; hCNT: human concentrative nucleoside transporters

of gemcitabine is inactivated mainly by CDA [Figure 2], that mediates conversion from gemcitabine to difluorodeoxyuridine $(\mathrm{dFdU})^{[38]}$. After deamination of gemcitabine, the metabolite is not further degraded but excreted from the cell ${ }^{[39]}$. CDA is activated in many organs, and $\mathrm{dFdU}$ is the major form of in vivo clearance which is the sole metabolite in the urine $e^{[40]}$. CDA is released from the cell and is found in the serum $^{[41]}$; CDA has been detected in patients with several cancer types and correlates with responses to chemotherapy ${ }^{[42,43]}$. The $C D A$ gene is affected by several genetic alterations, and marked variations in function ranging from null to increased activity have been observed ${ }^{[44]}$. A study conducted on pancreatic cancer patients with gemcitabine treatment demonstrated a correlation between CDA activity and chemoresistance and concluded that patients with $6 \mathrm{U} / \mathrm{mg}$ or higher of CDA activity showed progression of disease by five-fold or more ${ }^{[45]}$. A recent systemic review concludes that $C D A 79 \mathrm{~A}>\mathrm{C}$ polymorphism is a potential biomarker for toxicity of gemcitabine-based chemotherapy and that CDA testing is preferential before administration of gemcitabine ${ }^{[46]}$.

CDA upregulation decreases the cellular gemcitabine concentration [Figure 4], and several studies have reported that increased CDA activity associates with gemcitabine resistance in cancer cells. A hematopoietic cell line with overexpression of CDA showed resistance to gemcitabine (2.4-fold in $\mathrm{IC}_{50}$ and 2.5 -fold in $\left.\mathrm{IC}_{80}\right)^{[47]}$. On the other hand, studies using human tumour cell lines and tumour xenografts reported no association between chemoresistance and CDA activity ${ }^{[48,49]}$. These data showed that CDA is not the only determining factor for gemcitabine sensitivity in vivo, but its modulation may defeat chemoresistance.

In cancer cells, aberrations of the copy number of the $C D A$ gene are not reported. $C D A$ expression is mainly regulated transcriptionally and/or post-transcriptionally. $C D A$ expression in most cancers is lower than in corresponding normal tissues because of DNA methylation in the promoter region ${ }^{[50,51]}$. miRNAs also regulate CDA expression; miR-484 directly inhibits CDA translation by targeting CDA 3'UTR and induces chemoresistance in breast cancer cells ${ }^{[52]}$, and decreased expression of miR-608 correlates with upregulation of CDA to induce chemoresistance in pancreatic cancer cells ${ }^{[53]}$. Albumin-conjugated paclitaxel (nab-paclitaxel) was shown to reduce the CDA protein by producing reactive oxygen species in a mouse pancreatic cancer model; this evidence may explain the usefulness of gemcitabine plus nabpaclitaxel $(\mathrm{GnP})^{[54]}$.

Pharmacological inhibitors of CDA include zebularine, 3,4,5,6-tetrahydrouridine (THU) and 1, 3-diazepinone riboside (DR) [Figure 4]. Zebularine was first described in $1980^{[55]}$; it blocks CDA by way of a tetrahedral intermediate using the proton at $\mathrm{C} 4$ of pyrimidine ring ${ }^{[56]}$. However, zebularine also interacts with DNA methyltransferase; zebularine is not specific to $\mathrm{CDA}^{[57]}$. THU was first identified and purified 


\section{CDA inhibitors}

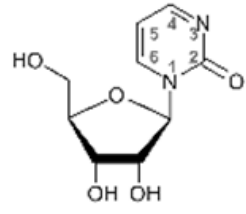

Zebularine
3,4,5,6-Tetrahydrouridine (THU)

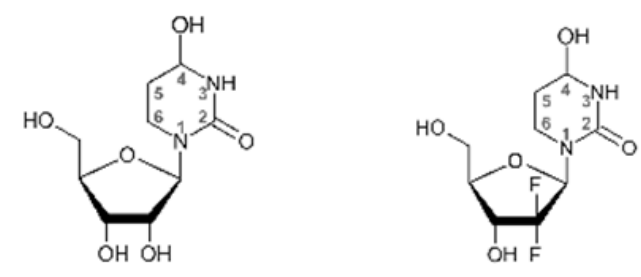

(4R)-2'-deoxy-2',2'-difluoro3, 4, 5, 6-tetrahydrouridine

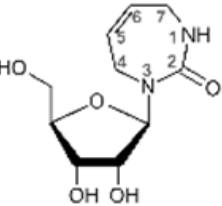

1,3-Diazepinone riboside (DR)

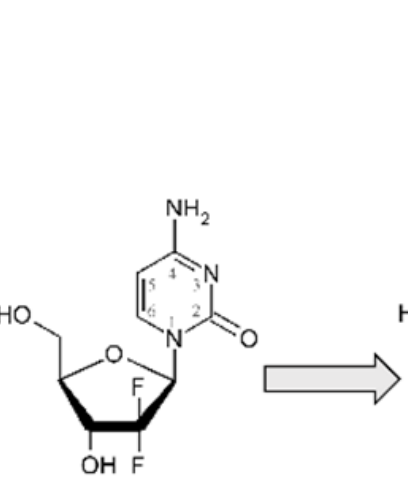

Gemcitabine

Activated $\perp$

\section{DNA synthesis}

Figure 4. CDA-mediated processing of gemcitabine for excretion, and CDA inhibitors Zebularine, 3,4,5,6-Tetrahydrouridine (THU), 1,3-Diazepinone riboside (DR), and (4R)-2'-deoxy-2',2'-difluoro-3 4, 5, 6-tetrahydrouridine. CDA: cytidine deaminase

in 1967 using an affinity capture method with CDA as bait ${ }^{[58]}$. The inhibitory action of THU is based on its C4 hydroxyl group in the pyrimidine ring. Since the bioavailability of THU is weak ${ }^{[59]}$, a new fluorinated version of this drug termed (4R)-2'-deoxy-2',2'w-difluoro-3 4, 5, 6-tetrahydrouridine [Figure 4] has been developed with better oral bioavailability ${ }^{[60]}$. DR was discovered in 1981; it cannot interact with CDA through the water/zinc complex. Its inhibitory activity instead results from an electrostatic interaction utilizing $\pi$ electrons of the DR ring and the benzene ring of the F137 of CDA, the catalytic site of the enzyme $^{[6]]}$. However, no results of DR effectiveness have yet been reported even in cultured cells.

As mentioned before, CDA high-expressing tumours are theoretically more resistant to cytidine-based therapies, including gemcitabine. With this assumption, several studies combining various chemotherapies and CDA inhibitors have been conducted to date. A Phase II clinical trial (ClinicalTraials.gov: NCT00978250, see Table 1), combining treatment with 5-fluoro-2'-deoxcytidine and THU, has just been completed; all 93 patients eligible for the study were assessed as PFS, including patients with advanced nonsmall cell lung cancer, breast cancer, bladder cancer, or head and neck cancer (https://www.clinicaltrials. gov/ct2/show/results/NCT00978250). Weizman et al. ${ }^{[62]}$ suggested that tumour infiltrating macrophages 
were responsible for stimulating the upregulation of CDA and acquisition of chemoresistance against gemcitabine in pancreatic cancer cells. Modulation of macrophage trafficking may offer a new strategy for response of cancer cells to gemcitabine ${ }^{[62,63]}$. Therefore, although CDA does not appear to be the only factor determining sensitivity to gemcitabine, its modulation remains a common strategy to overcome resistance.

\section{TRANSPORTERS INVOLVED IN EFFLUX OF GEMCITABINE AND ITS METABOLITES}

ATP-binding cassette $(\mathrm{ABC})$ transporters are known to translocate a wide variety of substrates across the cell membrane and to mediate resistance against many therapeutic drugs, including anti-neoplastics and anti-infectives ${ }^{[64]}$. In addition, $\mathrm{ABC}$ transporters associate with a fraction of stem-like cells called side population (SP), refractory to Hoechst 33342 dye staining. This subpopulation was first isolated from murine hematopoietic cells ${ }^{[65]}$ and then from human cells. Isolated SP cells from various kinds of human solid cancers escape from chemotherapy due to overexpression of the ABC transporters ${ }^{[66]}$, and Borst reviewed pan-resistance and $\mathrm{ABC}$ transporters ${ }^{[67]}$.

Several studies examining the importance of $A B C$-transporters in gemcitabine resistance have confirmed that the abnormal expression of $\mathrm{ABCB} 1, \mathrm{ABCC}$, and $\mathrm{ABCG} 2$ is associated with multidrug-resistance in pancreatic cancer ${ }^{[68]}$. On the other hand, MDR variants in two cell lines of small cell lung cancer showed increased DCK activity ${ }^{[69]}$, and human cancer cell lines overexpressing ABCB1 or ABCC1 showed increased sensitivity to gemcitabine ${ }^{[70]}$. Overexpression of ABCC4 and ABCC5 confer resistance to cytrabine and troxacitabine, but not gemcitabine ${ }^{[7]]}$. Inhibition of one or even several ABCC transporters (ABCC3, ABCC5 and ABCC10) did not efficiently or completely inhibit efflux of gemcitabine ${ }^{[72]}$. Thus, the contribution of $\mathrm{ABC}$ transporters for gemcitabine resistance warrants further investigation.

\section{PRODRUGS OF DCK FOR BYPASSING THE INTRACELLULAR PHOSPHORYLATION STEP}

Once gemcitabine is transported into cells, phosphorylation by DCK is considered to be the major rate-limiting factor for activation. DCK has a Km value of $4.6 \mu \mathrm{mol} / \mathrm{L}$ for gemcitabine compared to $1.5 \mu \mathrm{mol} / \mathrm{L}$ for deoxycytidine, which makes this drug an appropriate substrate ${ }^{[73]}$. Gemcitabine is also phosphorylated by thymidine kinase 2 . This is a mitochondrial enzyme which phosphorylates a broad range of natural nucleosides ${ }^{[74]}$, but its precise role for both gemcitabine host toxicity and anti-tumour activity is unclear ${ }^{[7]}$. Inactivation of DCK has been shown to be one of the key mechanisms for acquisition of gemcitabine resistance. The DCK gene is inactivated in all of the seven obtained gemcitabine-resistant cancer cell lines ${ }^{[75,76]}$. Knockdown of DCK leads to gemcitabine resistance in gemcitabine sensitive cell lines, while re-expression of $D C K$ restored the chemo-sensitivity of gemcitabine in gemcitabine-resistant cell lines ${ }^{[75,77,78]}$. Clinical studies have shown that the DCK expression level in pancreatic cancer tissue is a reliable prognostic indicator of PFS, suggesting that DCK is a good biomarker of gemcitabine sensitivity for pancreatic cancer patients treated with gemcitabine ${ }^{[79,80]}$. Hu antigen $\mathrm{R}(\mathrm{HuR})$ is an RNA-binding protein that regulates DCK post-transcriptionally. HuR is strongly associated with the DCK mRNA level, and HuRoverexpressing cancer cells have been shown to be more sensitive to gemcitabine treatment ${ }^{[81,82]}$.

Modification of phosphorylated gemcitabine to bypass DCK-mediated activation may be an effective way to improve its function. NUC-1031 [Figure $3 \mathrm{~B}$ ] is a gemcitabine phosphoramidate prodrug that is produced by ProTide Technology ${ }^{[83]}$. NUC-1031 enters into the cell independently of the hENT1 transporter and does not require activation by DCK. Similar to the phosphorylated forms of gemcitabine, NUC-1031 is not subject to breakdown by CDA. In a Phase I study (NCT01621854), NUC-1031 demonstrated clinically significant anti-tumour activity even in patients with prior gemcitabine exposure and in cancers not traditionally perceived as gemcitabine-responsive [Table 1] ${ }^{[84]}$. A global randomized study (NuTide:121) including 828 patients with untreated advanced biliary tract cancer is ongoing ${ }^{[85]}$. NUC-1031 is the first anti-cancer drug with which ProTide has achieved initial success in clinical trials. 
$\Delta$-Tocopherol-monophosphate gemcitabine (NUC050) is a vitamin E phosphate nucleoside prodrug [Figure $3 \mathrm{~B}$ ] designed to bypass two mechanisms of gemcitabine resistance: downregulation of hNTs, and downregulation of DCK. Incorporation of NUC050 is not affected by hNTs, suggesting that it can bypass them. NUC050 retains most of the activity in DCK deficient cells, indicating that gemcitabine monophosphate is delivered in the cell ${ }^{[86]}$.

Further formulation development will increase the safety and efficacy of these prodrugs to overcome the cancer chemoresistance induced by the down-regulation of DCK.

\section{RADIATION-INDUCED ACTIVATION OF DCK}

Most studies searching for synergism of radiation in combination with chemotherapeutic agents, including nucleoside analogues have been attempted to achieve radiosensitization of cancer cells. Gemcitabine is also employed clinically as a radiosensitizer ${ }^{[87]}$. The contribution of nucleoside analogues to synergic effects is thought to involve inhibition of DNA repair and modulation of nucleotide synthesis and availability. An alternative explanation for the synergism between radiation and nucleoside analogues is radiationmediated chemosensitization. A number of studies have demonstrated that radiation alone can enhance the activity of $\mathrm{DCK}^{[88-90]}$. One previous study showed that DCK is phosphorylated at S74 by the DNA damage responsive kinase ATM, and may be activated ${ }^{[91]}$; this indicates a direct link between radiation and DCK activation. Another study showed that the ATM related kinase ATR is also involved in phosphorylation of DCK at $574^{[92]}$. S74Q mutation of DCK increases $K_{\text {cat }}$ values by 11 -fold for deoxycytidine and 3-fold for gemcitabine $^{[93]}$. This in turn would explain the higher levels of active gemcitabine.

Recently, neoadjuvant therapy including radiation concurrent with gemcitabine has been conducted for borderline resectable pancreatic cancer ${ }^{[8]}$. Radiation may improve the cytotoxicity of gemcitabine by enhancing DCK activation.

\section{CONCLUSION}

Gemcitabine-based chemotherapy remains a cornerstone of treatment for patients with advanced cancers. Chemoresistance against gemcitabine is multifaceted; therefore, pursuing the improvement of this chemotherapy is still an important challenge. Novel methodologies are required to improve patients' prognoses.

In order to achieve an effective gemcitabine concentration within tumour cells, several considerations are needed. Nanoparticle-based medicine (nanomedicine) has numerous advantages compared with conventional medicines, including being able to protect gemcitabine from degradation, and provide a targeting delivery system. Some nanomedicines can accumulate inside tumour cells by the incorporation of ligands that target molecules overexpressed on the cancer cell surface ${ }^{[94]}$. Elechalawar et al.$^{[95]}$ developed a targeted drug delivery system to pancreatic cancer using gold nanoparticles as the delivery vehicle, the anti-EGFR antibody cetuximab (C225/C) as the targeting agent, gemcitabine as the effective drug, and polyethylene glycol (PEG) as the stealth molecule. This nanoconjugate, termed ACG44P1000, showed enhanced cellular uptake and cytotoxicity to pancreatic cancer cell lines in vitro study. Although the effect of this nanoconjugate may be limited, further investigations will lead to more effective improvements.

Tumours are heterogeneous and exhibit molecular complexity, with significant variation among patients. Treatments of cancer patients require precision medicine-based genetic and biomolecular characteristics. The traditional chemotherapeutic approach (one-size-fits-all) can lead to unnecessary exposure to adverse side effects without the anticipated survival benefits ${ }^{[96]}$. In the last decade, improvements in highthroughput sequencing methods and profiling of transcripts have led to the discovery of many new targets 
for treatments. The identification of receptor overexpression in cancer cells will lead to the development of nanomedicines to improve the selectivity to the cancer cells and reduce off-target toxicities of gemcitabine. Further studies are needed for gemcitabine-based treatment to be included in personalized medicine tailored for numerous molecular therapeutic targets in multiple pathogenic pathways.

\section{DECLARATIONS}

\section{Acknowledgments}

We are grateful to Barbara Lee Smith Pierce (retired Adjunct Professor, University of Maryland University College) for her editorial work in the preparation of the manuscript.

\section{Authors' contributions}

Made substantial contributions to conception of the review: Saiki Y, Horii A

Wrote the first draft of the manuscript: Saiki Y

Prepared tables and figures: Saiki Y, Hirota S

Brushed up the manuscript, tables, and figures: Saiki Y, Hirota S, Horii A

\section{Availability of data and materials}

Not applicable.

\section{Financial support and sponsorship}

None.

\section{Conflicts of interest}

All authors declared that there are no conflicts of interest.

\section{Ethical approval and consent to participate}

Not applicable.

\section{Consent for publication}

Not applicable.

\section{Copyright}

(c) The Author(s) 2020.

\section{REFERENCES}

1. Bonjouklian R, Phillips ML, Kuhler KM, Grindey GB, Poore GA, et al. Studies of the antitumor activity of (2-alkoxyalkyl)- and (2-alkoxyalkenyl)phosphocholines. J Med Chem 1986;29:2472-7.

2. Heinemann V, Hertel LW, Grindey GB, Plunkett W. Comparison of the cellular pharmacokinetics and toxicity of 2',2'-difluorodeoxycytidine and 1-beta-D-arabinofuranosylcytosine. Cancer Res 1988;48:4024-31.

3. Boswell-Casteel RC, Hays FA. Equilibrative nucleoside transporters-a review. Nucleosides Nucleotides Nucleic Acids 2017;36:7-30.

4. Shimada T, Nakanishi T, Tajima H, Yamazaki M, Yokono R, et al. Saturable hepatic extraction of gemcitabine involves biphasic uptake mediated by nucleoside transporters Equilibrative Nucleoside Transporter 1 and 2. J Pharm Sci 2015;104:3162-9.

5. Mini E, Nobili S, Caciagli B, Landini I, Mazzei T. Cellular pharmacology of gemcitabine. Ann Oncol 2006;17 Suppl 5:v7-12.

6. Ruiz van Haperen VW, Veerman G, Vermorken JB, Peters GJ. 2',2'-Difluoro-deoxycytidine (gemcitabine) incorporation into RNA and DNA of tumour cell lines. Biochem Pharmacol 1993;46: 762-6.

7. Bergman AM, Pinedo HM, Peters GJ. Determinants of resistance to 2',2'-difluorodeoxycytidine (gemcitabine). Drug Resist Updat 2002;5:19-33.

8. Kroep JR, Giaccone G, Tolis C, Voorn DA, Loves WJ, et al. Sequence dependent effect of paclitaxel on gemcitabine metabolism in relation to cell cycle and cytotoxicity in non-small-cell lung cancer cell lines. Br J Cancer 2000;83:1069-76.

9. Honeywell RJ, Ruiz van Haperen VW, Veerman G, Smid K, Peters GJ. Inhibition of thymidylate synthase by 2',2'-difluoro-2'deoxycytidine (Gemcitabine) and its metabolite 2',2'-difluoro-2'-deoxyuridine. Int J Biochem Cell Biol 2015;60:73-81.

10. Motoi F, Unno M. Neoadjuvant treatment for resectable pancreatic adenocarcinoma: What is the best protocol? Ann Gastroenterol Surg 
2020;4:100-8.

11. Gesto DS, Cerqueira NM, Fernandes PA, Ramos MJ. Gemcitabine: a critical nucleoside for cancer therapy. Curr Med Chem 2012;19:1076-87.

12. Kelderman S, Schumacher TN, Haanen JB. Acquired and intrinsic resistance in cancer immunotherapy. Mol Oncol 2014;8:1132-9.

13. Zheng X, Carstens JL, Kim J, Scheible M, Kaye J, et al. Epithelial-to-mesenchymal transition is dispensable for metastasis but induces chemoresistance in pancreatic cancer. Nature 2015;527:525-30.

14. Arumugam T, Ramachandran V, Fournier KF, Wang H, Marquis L, et al. Epithelial to mesenchymal transition contributes to drug resistance in pancreatic cancer. Cancer Res 2009;69:5820-8.

15. Fang Y, Zhou W, Rong Y, Kuang T, Xu X, et al. Exosomal miRNA-106b from cancer-associated fibroblast promotes gemcitabine resistance in pancreatic cancer. Exp Cell Res 2019;383:111543.

16. Yang Z, Zhao N, Cui J, Wu H, Xiong J, et al. Exosomes derived from cancer stem cells of gemcitabine-resistant pancreatic cancer cells enhance drug resistance by delivering miR-210. Cell Oncol (Dordr) 2020;43:123-36.

17. Ritzel MW, Ng AM, Yao SY, Graham K, Loewen SK, et al. Recent molecular advances in studies of the concentrative Na+-dependent nucleoside transporter (CNT) family: identification and characterization of novel human and mouse proteins (hCNT3 and mCNT3) broadly selective for purine and pyrimidine nucleosides (system cib). Mol Membr Biol 2001;18:65-72.

18. Lostao MP, Mata JF, Larrayoz IM, Inzillo SM, Casado FJ, et al. Electrogenic uptake of nucleosides and nucleoside-derived drugs by the human nucleoside transporter 1 (hCNT1) expressed in Xenopus laevis oocytes. FEBS Lett 2000;481:137-40.

19. Mackey JR, Mani RS, Selner M, Mowles D, Young JD, et al. Functional nucleoside transporters are required for gemcitabine influx and manifestation of toxicity in cancer cell lines. Cancer Res 1998;58:4349-57.

20. Garcia-Manteiga J, Molina-Arcas M, Casado FJ, Mazo A, Pastor-Anglada M. Nucleoside transporter profiles in human pancreatic cancer cells: Role of hCNT1 in 2',2'-difluorodeoxycytidine- induced cytotoxicity. Clin Cancer Res 2003;9:5000-5008.

21. Rauchwerger DR, Firby PS, Hedley DW, Moore MJ. Equilibrative-sensitive nucleoside transporter and its role in gemcitabine sensitivity. Cancer Res 2000;60:6075-9.

22. Mohelnikova-Duchonova B, Melichar B. Human equilibrative nucleoside transporter 1 (hENT1): do we really have a new predictive biomarker of chemotherapy outcome in pancreatic cancer patients? Pancreatology 2013;13:558-63.

23. Orlandi A, Calegari MA, Martini M, Cocomazzi A, Bagala C, et al. Gemcitabine versus FOLFIRINOX in patients with advanced pancreatic adenocarcinoma hENT1-positive: everything was not too bad back when everything seemed worse. Clin Transl Oncol 2016;18:988-95.

24. Bird NT, Elmasry M, Jones R, Psarelli E, Dodd J, et al. Immunohistochemical hENT1 expression as a prognostic biomarker in patients with resected pancreatic ductal adenocarcinoma undergoing adjuvant gemcitabine-based chemotherapy. Br J Surg 2017;104:328-36.

25. Nishio R, Tsuchiya H, Yasui T, Matsuura S, Kanki K, et al. Disrupted plasma membrane localization of equilibrative nucleoside transporter 2 in the chemoresistance of human pancreatic cells to gemcitabine (dFdCyd). Cancer Sci 2011;102:622-9.

26. Mohelnikova-Duchonova B, Brynychova V, Oliverius M, Honsova E, Kala Z, et al. Differences in transcript levels of ABC transporters between pancreatic adenocarcinoma and nonneoplastic tissues. Pancreas 2013;42:707-16.

27. Bhutia YD, Hung SW, Patel B, Lovin D, Govindarajan R. CNT1 expression influences proliferation and chemosensitivity in drugresistant pancreatic cancer cells. Cancer Res 2011;71:1825-35.

28. Skrypek N, Duchene B, Hebbar M, Leteurtre E, van Seuningen I, et al. The MUC4 mucin mediates gemcitabine resistance of human pancreatic cancer cells via the Concentrative Nucleoside Transporter family. Oncogene 2013;32:1714-23.

29. Chen P, Chien PY, Khan AR, Sheikh S, Ali SM, et al. In vitro and in vivo anti-cancer activity of a novel gemcitabine-cardiolipin conjugate. Anticancer Drugs 2006;17:53-61.

30. Bergman AM, Adema AD, Balzarini J, Bruheim S, Fichtner I, et al. Antiproliferative activity, mechanism of action and oral antitumor activity of CP-4126, a fatty acid derivative of gemcitabine, in in vitro and in vivo tumor models. Invest New Drugs 2011;29:456-66.

31. Poplin E, Wasan H, Rolfe L, Raponi M, Ikdahl T, et al. Randomized, multicenter, phase II study of CO-101 versus gemcitabine in patients with metastatic pancreatic ductal adenocarcinoma: including a prospective evaluation of the role of hENT1 in gemcitabine or CO-101 sensitivity. J Clin Oncol 2013;31:4453-61.

32. Raffenne J, Nicolle R, Puleo F, Le Corre D, Boyez C, et al. hENT1 testing in pancreatic ductal adenocarcinoma: are we ready? a multimodal evaluation of hENT1 status. Cancers (Basel) 2019;11.

33. Guo Z, Wang F, Di Y, Yao L, Yu X, et al. Antitumor effect of gemcitabine-loaded albumin nanoparticle on gemcitabine-resistant pancreatic cancer induced by low hENT1 expression. Int J Nanomedicine 2018;13:4869-80.

34. Couvreur P, Stella B, Reddy LH, Hillaireau H, Dubernet C, et al. Squalenoyl nanomedicines as potential therapeutics. Nano Lett 2006;6:2544-8.

35. Bildstein L, Dubernet C, Marsaud V, Chacun H, Nicolas V, et al. Transmembrane diffusion of gemcitabine by a nanoparticulate squalenoyl prodrug: an original drug delivery pathway. J Control Release 2010;147:163-70.

36. Chitkara D, Mittal A, Behrman SW, Kumar N, Mahato RI. Self-assembling, amphiphilic polymer-gemcitabine conjugate shows enhanced antitumor efficacy against human pancreatic adenocarcinoma. Bioconjug Chem 2013;24:1161-73.

37. Wonganan P, Lansakara PD, Zhu S, Holzer M, Sandoval MA, et al. Just getting into cells is not enough: mechanisms underlying 4-(N)stearoyl gemcitabine solid lipid nanoparticle's ability to overcome gemcitabine resistance caused by RRM1 overexpression. J Control Release 2013;169:17-27.

38. Frances A, Cordelier P. The emerging role of cytidine deaminase in human diseases: a new opportunity for therapy? Mol Ther 2020;28:357-66. 
39. Heinemann V, Xu YZ, Chubb S, Sen A, Hertel LW, et al. Cellular elimination of 2',2'-difluorodeoxycytidine 5'-triphosphate: a mechanism of self-potentiation. Cancer Res 1992;52:533-9.

40. Abbruzzese JL, Grunewald R, Weeks EA, Gravel D, Adams T, et al. A phase I clinical, plasma, and cellular pharmacology study of gemcitabine. J Clin Oncol 1991;9:491-8.

41. Chalmers IM, Thomson GT, Desjardins P. Serum cytidine deaminase as a laboratory test for acute inflammation in rheumatoid arthritis. Ann Rheum Dis 1988;47:173-4.

42. Peters GJ, Honeywell RJ, Maulandi M, Giovannetti E, Losekoot N, et al. Selection of the best blood compartment to measure cytidine deaminase activity to stratify for optimal gemcitabine or cytarabine treatment. Nucleosides Nucleotides Nucleic Acids 2014;33:403-12.

43. Sugiyama E, Kaniwa N, Kim SR, Hasegawa R, Saito Y, et al. Population pharmacokinetics of gemcitabine and its metabolite in Japanese cancer patients: impact of genetic polymorphisms. Clin Pharmacokinet 2010;49:549-58.

44. Micozzi D, Carpi FM, Pucciarelli S, Polzonetti V, Polidori P, et al. Human cytidine deaminase: a biochemical characterization of its naturally occurring variants. Int J Biol Macromol 2014;63:64-74.

45. Serdjebi C, Seitz JF, Ciccolini J, Duluc M, Norguet E, et al. Rapid deaminator status is associated with poor clinical outcome in pancreatic cancer patients treated with a gemcitabine-based regimen. Pharmacogenomics 2013;14:1047-51.

46. Ding X, Chen W, Fan H, Zhu B. Cytidine deaminase polymorphism predicts toxicity of gemcitabine-based chemotherapy. Gene 2015;559:31-7.

47. Neff T, Blau CA. Forced expression of cytidine deaminase confers resistance to cytosine arabinoside and gemcitabine. Exp Hematol 1996;24:1340-6.

48. van Haperen VW, Veerman G, Vermorken JB, Pinedo HM, Peters G. Regulation of phosphorylation of deoxycytidine and 2',2'-difluorodeoxycytidine (gemcitabine); effects of cytidine 5'-triphosphate and uridine 5'-triphosphate in relation to chemosensitivity for 2',2'-difluorodeoxycytidine. Biochem Pharmacol 1996;51:911-8.

49. Ruiz van Haperen VW, Veerman G, Braakhuis BJ, Vermorken JB, Boven E, et al. Deoxycytidine kinase and deoxycytidine deaminase activities in human tumour xenografts. Eur J Cancer 1993;29A:2132-7.

50. Mameri H, Bieche I, Meseure D, Marangoni E, Buhagiar-Labarchede G, et al. Cytidine deaminase deficiency reveals new therapeutic opportunities against cancer. Clin Cancer Res 2017;23:2116-26.

51. Zauri M, Berridge G, Thezenas ML, Pugh KM, Goldin R, et al. CDA directs metabolism of epigenetic nucleosides revealing a therapeutic window in cancer. Nature 2015;524:114-8.

52. Ye FG, Song CG, Cao ZG, Xia C, Chen DN, et al. Cytidine deaminase axis modulated by miR-484 differentially regulates cell proliferation and chemoresistance in breast cancer. Cancer Res 2015;75:1504-15.

53. Rajabpour A, Afgar A, Mahmoodzadeh H, Radfar JE, Rajaei F, et al. MiR-608 regulating the expression of ribonucleotide reductase M1 and cytidine deaminase is repressed through induced gemcitabine chemoresistance in pancreatic cancer cells. Cancer Chemother Pharmacol 2017;80:765-75.

54. Frese KK, Neesse A, Cook N, Bapiro TE, Lolkema MP, et al. nab-Paclitaxel potentiates gemcitabine activity by reducing cytidine deaminase levels in a mouse model of pancreatic cancer. Cancer Discov 2012;2:260-9.

55. McCormack JJ, Marquez VE, Liu PS, Vistica DT, Driscoll JS. Inhibition of cytidine deaminase by 2-oxopyrimidine riboside and related compounds. Biochem Pharmacol 1980;29:830-2.

56. Betts L, Xiang S, Short SA, Wolfenden R, Carter CW Jr. Cytidine deaminase. The 2.3 A crystal structure of an enzyme: transition-state analog complex. J Mol Biol 1994;235:635-56.

57. Zhou L, Cheng X, Connolly BA, Dickman MJ, Hurd PJ, et al. Zebularine: a novel DNA methylation inhibitor that forms a covalent complex with DNA methyltransferases. J Mol Biol 2002;321:591-9.

58. Hanze AR. Nucleic acids. IV. The catalytic reduction of pyrimidine nucleosides (human liver deaminase inhibitors). J Am Chem Soc 1967;89:6720-5.

59. Beumer JH, Eiseman JL, Gilbert JA, Holleran JL, Yellow-Duke AE, et al. Plasma pharmacokinetics and oral bioavailability of the 3,4,5,6-tetrahydrouridine (THU) prodrug, triacetyl-THU (taTHU), in mice. Cancer Chemother Pharmacol 2011;67:421-30.

60. Ferraris D, Duvall B, Delahanty G, Mistry B, Alt J, et al. Design, synthesis, and pharmacological evaluation of fluorinated tetrahydrouridine derivatives as inhibitors of cytidine deaminase. J Med Chem 2014;57:2582-8.

61. Chung SJ, Fromme JC, Verdine GL. Structure of human cytidine deaminase bound to a potent inhibitor. J Med Chem 2005;48:658-60.

62. Weizman N, Krelin Y, Shabtay-Orbach A, Amit M, Binenbaum Y, et al. Macrophages mediate gemcitabine resistance of pancreatic adenocarcinoma by upregulating cytidine deaminase. Oncogene 2014;33:3812-9.

63. Amit M, Gil Z. Macrophages increase the resistance of pancreatic adenocarcinoma cells to gemcitabine by upregulating cytidine deaminase. Oncoimmunology 2013;2:e27231.

64. Eckford PD, Sharom FJ. ABC efflux pump-based resistance to chemotherapy drugs. Chem Rev 2009;109:2989-3011.

65. Goodell MA, Rosenzweig M, Kim H, Marks DF, DeMaria M, et al. Dye efflux studies suggest that hematopoietic stem cells expressing low or undetectable levels of CD34 antigen exist in multiple species. Nat Med 1997;3:1337-45.

66. Fukuda K, Saikawa Y, Ohashi M, Kumagai K, Kitajima M, et al. Tumor initiating potential of side population cells in human gastric cancer. Int J Oncol 2009;34:1201-7.

67. Borst P. Cancer drug pan-resistance: pumps, cancer stem cells, quiescence, epithelial to mesenchymal transition, blocked cell death pathways, persisters or what? Open Biol 2012;2:120066.

68. Polgar O, Bates SE. ABC transporters in the balance: is there a role in multidrug resistance? Biochem Soc Trans 2005;33:241-5.

69. Bergman AM, Munch-Petersen B, Jensen PB, Sehested M, Veerman G, et al. Collateral sensitivity to gemcitabine 
(2',2'-difluorodeoxycytidine) and cytosine arabinoside of daunorubicin- and VM-26-resistant variants of human small cell lung cancer cell lines. Biochem Pharmacol 2001;61:1401-8.

70. Bergman AM, Pinedo HM, Talianidis I, Veerman G, Loves WJ, et al. Increased sensitivity to gemcitabine of P-glycoprotein and multidrug resistance-associated protein-overexpressing human cancer cell lines. Br J Cancer 2003;88:1963-70.

71. Adema AD, Floor K, Smid K, Honeywell RJ, Scheffer GL, et al. Overexpression of MRP4 (ABCC4) and MRP5 (ABCC5) confer resistance to the nucleoside analogs cytarabine and troxacitabine, but not gemcitabine. Springerplus 2014;3:732.

72. Rudin D, Li L, Niu N, Kalari KR, Gilbert JA, et al. Gemcitabine cytotoxicity: interaction of efflux and deamination. J Drug Metab Toxicol 2011;2:1-10.

73. Bouffard DY, Laliberte J, Momparler RL. Kinetic studies on 2',2'-difluorodeoxycytidine (Gemcitabine) with purified human deoxycytidine kinase and cytidine deaminase. Biochem Pharmacol 1993;45:1857-61.

74. Wang L, Munch-Petersen B, Herrstrom Sjoberg A, Hellman U, Bergman T, et al. Human thymidine kinase 2: molecular cloning and characterisation of the enzyme activity with antiviral and cytostatic nucleoside substrates. FEBS Lett 1999;443:170-4.

75. Saiki Y, Yoshino Y, Fujimura H, Manabe T, Kudo Y, et al. DCK is frequently inactivated in acquired gemcitabine-resistant human cancer cells. Biochem Biophys Res Commun 2012;421:98-104.

76. Nakano T, Saiki Y, Kudo C, Hirayama A, Mizuguchi Y, et al. Acquisition of chemoresistance to gemcitabine is induced by a loss-offunction missense mutation of DCK. Biochem Biophys Res Commun 2015;464:1084-9.

77. Tang K, Zhang Z, Bai Z, Ma X, Guo W, et al. Enhancement of gemcitabine sensitivity in pancreatic cancer by co-regulation of dCK and p8 expression. Oncol Rep 2011;25:963-70.

78. Funamizu N, Okamoto A, Kamata Y, Misawa T, Uwagawa T, et al. Is the resistance of gemcitabine for pancreatic cancer settled only by overexpression of deoxycytidine kinase? Oncol Rep 2010;23:471-5.

79. Sierzega M, Pach R, Kulig P, Legutko J, Kulig J. Prognostic implications of expression profiling for gemcitabine-related genes (hENT1, dCK, RRM1, RRM2) in patients with resectable pancreatic adenocarcinoma receiving adjuvant chemotherapy. Pancreas 2017;46:684-9.

80. Ohmine K, Kawaguchi K, Ohtsuki S, Motoi F, Ohtsuka H, et al. Quantitative targeted proteomics of pancreatic cancer: Deoxycytidine kinase protein level correlates to progression-free survival of patients receiving gemcitabine treatment. Mol Pharm 2015;12:3282-91.

81. Costantino CL, Witkiewicz AK, Kuwano Y, Cozzitorto JA, Kennedy EP, et al. The role of HuR in gemcitabine efficacy in pancreatic cancer: HuR Up-regulates the expression of the gemcitabine metabolizing enzyme deoxycytidine kinase. Cancer Res 2009;69:4567-72.

82. Tatarian T, Jiang W, Leiby BE, Grigoli A, Jimbo M, et al. Cytoplasmic HuR status predicts disease-free survival in resected pancreatic cancer: a post-hoc analysis from the International Phase III ESPAC-3 Clinical Trial. Ann Surg 2018;267:364-9.

83. Slusarczyk M, Lopez MH, Balzarini J, Mason M, Jiang WG, et al. Application of ProTide technology to gemcitabine: a successful approach to overcome the key cancer resistance mechanisms leads to a new agent (NUC-1031) in clinical development. J Med Chem 2014;57:1531-42.

84. Blagden SP, Rizzuto I, Suppiah P, O’Shea D, Patel M, et al. Anti-tumour activity of a first-in-class agent NUC-1031 in patients with advanced cancer: results of a phase I study. Br J Cancer 2018;119:815-22.

85. McNamara MG, Goyal L, Doherty M, Springfeld C, Cosgrove D, et al. NUC-1031/cisplatin versus gemcitabine/cisplatin in untreated locally advanced/metastatic biliary tract cancer (NuTide:121). Future Oncol 2020; doi: 10.2217/fon-2020-0247.

86. Daifuku R, Koratich M, Stackhouse M. Vitamin E phosphate nucleoside prodrugs: a platform for intracellular delivery of monophosphorylated nucleosides. Pharmaceuticals (Basel) 2018;11.

87. Shewach DS, Hahn TM, Chang E, Hertel LW, Lawrence TS. Metabolism of 2',2'-difluoro-2'-deoxycytidine and radiation sensitization of human colon carcinoma cells. Cancer Res 1994;54:3218-23.

88. Gregoire V, Rosier JF, De Bast M, Bruniaux M, De Coster B, et al. Role of deoxycytidine kinase (dCK) activity in gemcitabine's radioenhancement in mice and human cell lines in vitro. Radiother Oncol 2002;63:329-38.

89. Van Den Neste E, Smal C, Cardoen S, Delacauw A, Frankard J, et al. Activation of deoxycytidine kinase by UV-C-irradiation in chronic lymphocytic leukemia B-lymphocytes. Biochem Pharmacol 2003;65:573-80.

90. Sigmond J, Haveman J, Kreder NC, Loves WJ, van Bree C, et al. Enhanced activity of deoxycytidine kinase after pulsed low dose rate and single dose gamma irradiation. Nucleosides Nucleotides Nucleic Acids 2006;25:1177-80.

91. Yang C, Lee M, Hao J, Cui X, Guo X, et al. Deoxycytidine kinase regulates the G2/M checkpoint through interaction with cyclindependent kinase 1 in response to DNA damage. Nucleic Acids Res 2012;40:9621-32.

92. Beyaert M, Starczewska E, Van Den Neste E, Bontemps F. A crucial role for ATR in the regulation of deoxycytidine kinase activity. Biochem Pharmacol 2016;100:40-50.

93. McSorley T, Ort S, Hazra S, Lavie A, Konrad M. Mimicking phosphorylation of Ser-74 on human deoxycytidine kinase selectively increases catalytic activity for $\mathrm{dC}$ and $\mathrm{dC}$ analogues. FEBS Lett 2008;582:720-4.

94. Sharma B, Crist RM, Adiseshaiah PP. Nanotechnology as a delivery tool for precision cancer therapies. AAPS J 2017;19:1632-42.

95. Elechalawar CK, Hossen MN, Shankarappa P, Peer CJ, Figg WD, et al. Targeting pancreatic cancer cells and stellate cells using designer nanotherapeutics in vitro. Int J Nanomedicine 2020;15:991-1003.

96. Torres C, Grippo PJ. Pancreatic cancer subtypes: a roadmap for precision medicine. Ann Med 2018;50:277-87. 